\title{
Avaliação dinâmica e interdisciplinar na deficiência auditiva em ambulatório público: relato de caso
}

\section{Dynamic and interdisciplinary assessment in hearing disability in a public ambulatory: case report}

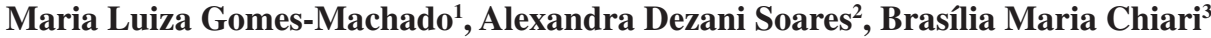

\begin{abstract}
RESUMO
A proposta deste estudo foi descrever os efeitos da avaliação clínica dinâmica interdisciplinar em um caso atendido pelo Ambulatório de Investigação da Linguagem nos Distúrbios de Audição do Hospital São Paulo - Universidade Federal de São Paulo. Trata-se de uma pessoa do sexo feminino que, aos 26 anos, apresentou quadro de surdez súbita bilateral, em decorrência da síndrome de Cogan - que afeta também a visão. Ao iniciar o processo, a paciente apresentava leitura orofacial restrita, dificuldade para se comunicar com sua família, isolamento social e quadro depressivo. A avaliação dinâmica pautada na interatividade e na escuta humanística proporcionou condições para que a paciente pudesse melhorar sua comunicação, autoconceito e interação social. Nessa perspectiva, observou-se que, com apenas dez sessões de atendimento pela equipe, operaram-se efeitos terapêuticos quebrando-se, assim, a clássica dicotomia avaliação/intervenção em momentos diferentes.
\end{abstract}

Descritores: Surdez; Reabilitação de deficientes auditivos; Linguagem; Equipe de assistência ao paciente; Relatos de casos

\section{INTRODUÇÃO}

Deficiência auditiva, conforme o artigo 70, capítulo IX, das disposições finais do Decreto ${ }^{0}$ 5.296, de 2004, pode ser definida como perda bilateral, parcial ou total de $41 \mathrm{~dB}$ ou mais da audição, aferida por audiograma nas frequências de $500 \mathrm{~Hz}$, $1000 \mathrm{~Hz}, 2000 \mathrm{~Hz}$ e $3000 \mathrm{~Hz}^{(1)}$. Existem diversos tipos e graus de deficiência auditiva, com diferentes etiologias. Em geral, as pessoas com perdas auditivas de grau leve e moderado são chamadas de deficientes auditivas, enquanto as que possuem perdas severas (entre 70 e $90 \mathrm{~dB}$ ) e profundas (acima de 90 dB) são consideradas surdas.

A perda auditiva pode ser percebida logo nos primeiros anos de vida, pelos pais ou profissionais que trabalham com a

Trabalho realizado no Departamento de Fonoaudiologia da Universidade Federal de São Paulo - UNIFESP - São Paulo (SP), Brasil.

(1) Pós-graduanda (Doutorado) pela Universidade Federal de São Paulo UNIFESP - São Paulo (SP), Brasil; Psicóloga da Associação de Pais e Amigos dos Excepcionais - APAE - São Paulo (SP), Brasil.

(2) Mestre, Fonoaudióloga do Centro de Deficiente Auditivo da Universidade Federal de São Paulo - UNIFESP - São Paulo (SP), Brasil; Fonoaudióloga da Fundação para o Estudo e Tratamento das Deformidades Crânio Faciais - FUNCRAF - Grande São Paulo (SP), Brasil.

(3) Professora titular da Disciplina de Distúrbios da Comunicação Humana do Departamento de Fonoaudiologia da Universidade Federal de São Paulo - UNIFESP - São Paulo (SP), Brasil.

Endereço para correspondência: Maria Luiza Gomes-Machado. R. Agostinho Rodrigues Filho, 410/81, Vila Clementino, São Paulo (SP), Brasil, CEP: 04026-040. E-mail: malugmachado@ hotmail.com

Recebido em: 27/6/2008; Aceito em: 2/12/2008 criança. O diagnóstico envolve avaliação médica e audiológica, bem como orientação criteriosa à família e à pessoa acometida pela deficiência.

Quanto mais cedo for diagnosticada a deficiência, maiores as possibilidades de intervenção e auxílio no desenvolvimento sociocognitivo da pessoa, visto que a audição é essencial à organização do pensamento, à compreensão do mundo e do outro, fazendo parte de uma das funções superiores mais nobres do ser humano, que é a comunicação ${ }^{(2)}$.

O cuidado com a audição deve ser constante, pois algumas doenças podem afetar a capacidade auditiva no decorrer da vida, entre elas a síndrome de Cogan, apresentada pela paciente de nosso relato de caso. Essa síndrome foi relatada por David Cogan, em $1945^{*}$, e se refere a um distúrbio imunológico raro, que acomete predominantemente jovens adultos. Os sintomas envolvem inflamação ocular recorrente e disfunção auditivovestibular, levando à perda da audição em poucos meses, podendo vir a ser uma surdez permanente, caso o diagnóstico seja $\operatorname{tardio}^{(3)}$. Daí a importância da cuidadosa investigação clínica.

Na esfera da Fonoaudiologia, a avaliação clínica é considerada essencial à prevenção de comorbidades e à reabilitação, pressupondo aspectos técnicos ligados à comunicação, mas, sobretudo, uma concepção humanística de atendimento, que

\footnotetext{
* Cogan DG. Syndrome of nonsyphilitis interstitial keratitis and vestibuloauditory symptoms. Arch Ophthalmol. 1945;33:144-9 apud St Clair EW, McCallum R. Cogan's Syndrome. In: Hochberg MC, Silman AJ, Smolen JS, Weinblatt ME, Weisman MH. Rheumatology. 4th ed. Philadelphia: MosbyElsevier; 2008.
} 
envolva uma equipe interdisciplinar voltada à compreensão do sujeito em seu contexto socioambiental ${ }^{(4)}$.

$\mathrm{O}$ desenvolvimento da linguagem e do pensamento implica processos complexos de interação entre o indivíduo e o ambiente, de tal forma que as interferências externas possam afetar, de forma positiva ou negativa, toda a sua evolução ${ }^{(5)}$.

Na primeira metade do século XX, foram desenvolvidas teorias que relacionavam os processos psicológicos aos aspectos históricos e culturais, enfatizando o papel fundamental da linguagem no desenvolvimento humano ${ }^{(5-6)}$. A língua funciona não apenas como uma forma de comunicação, mas também como função reguladora do pensamento. Pensar envolve todo o sistema de percepção dos estímulos ambientais. E, nesse contexto, a audição tem função capital ${ }^{(6)}$.

Estudos demonstram que o ambiente sociocultural no qual a pessoa se desenvolve, sobretudo a interação afetiva, tem implicações relevantes para a recepção e o processamento das informações. Nessa ótica, o processo cognitivo se opera a partir da linguagem, havendo um relacionamento intrínseco entre linguagem e interação social ${ }^{(7)}$.

Nessa abordagem, podemos dizer que, durante a avaliação clínica interdisciplinar do paciente com deficiência auditiva, se torna imprescindível considerar as dimensões socioculturais de cada sujeito avaliado.

Por equipe interdisciplinar, na área da saúde, entendese o trabalho de profissionais de diferentes disciplinas, que atuam de forma planejada e integrada, no esforço comum de maximizar a recuperação do paciente e da família. Cada profissional, embora ciente de sua função e do papel dos demais, compartilha o seu conhecimento visando o melhor atendimento do paciente ${ }^{(8)}$.

Nessa abordagem, a Fonoaudiologia pode colaborar para a identificação das prioridades e necessidades de comunicação do paciente, estabelecendo metas que contribuam para a diminuição das defasagens comunicativas, proporcionando-lhe melhor qualidade de vida ${ }^{(9)}$.

No âmbito da Psicologia, o diagnóstico da pessoa com deficiência deve ultrapassar o antigo enfoque médico/pedagógico, não se fundamentando exclusivamente em traços únicos, valorizando, assim, as potencialidades do sujeito dentro da comunidade em que vive ${ }^{(10)}$.

Um fator importante, a ser considerado no processo diagnóstico da pessoa com deficiência auditiva, refere-se à avaliação do autoconceito, pois esse aspecto é de suma relevância para a adaptabilidade do sujeito ao uso de aparelho auditivo(11). A família e o grupo social são imprescindíveis para a construção da identidade do sujeito: a percepção do outro ou as representações do grupo social funcionarão como referenciais para a formação do autoconceito ${ }^{(12)}$. Logo, a avaliação diagnóstica não pode abstrair de criteriosa observação e consideração do meio familiar, escolar e comunitário em que o sujeito vive, visando às melhores formas de adaptação e inclusão.

A avaliação não deve ser estanque, restringindo-se a mera descrição dos fenômenos e sintomas. Na abordagem diagnóstica dinâmica, inspirada nos pressupostos de Vygotsky, considera-se a interação entre os profissionais e o paciente, centrando-se na identificação do desempenho potencial deste e nas intervenções que possam favorecer um avanço no desenvolvimento. Portanto, durante a avaliação, o mediador fornece pistas, instruções, demonstrações, orientações e estímulos, apostando em mudanças no quadro durante o processo diagnóstico ${ }^{(13)}$. Saber ter escuta ativa diante do discurso do paciente, como relata o autor ${ }^{(14)}$, o impele a uma fala que por si só faz com que os conflitos possam emergir. Por outro lado, também se sabe que é relevante oferecer ao paciente um espaço no qual ele possa se colocar de forma espontânea, sentindo-se acolhido em um atendimento mais humanizado ${ }^{(15)}$.

Nesta perspectiva, este estudo visa apresentar o caso clínico de uma paciente, Márcia**, com diagnóstico de síndrome de Cogan, avaliada por uma equipe interdisciplinar do Ambulatório de Investigação nos Distúrbios de Audição do Hospital São Paulo - Universidade Federal de São Paulo (UNIFESP), com o intuito de traçarmos algumas considerações acerca do processo diagnóstico sob um enfoque dinâmico-terapêutico.

\section{APRESENTAÇÃO DO CASO CLÍNICO}

O estudo foi realizado no Ambulatório de Investigação da Linguagem nos Distúrbios de Audição, que oferece atendimento ao público por meio da atuação de estagiários, especialistas, mestrandos e doutorandos, sob a supervisão e orientação de professor titular, livre-docente na área de Fonoaudiologia. Dentre os inúmeros casos que passam pelo Ambulatório e se beneficiam da visão interdisciplinar no processo de avaliação, optamos por apresentar o caso de Márcia, dadas a complexidade e a gravidade do problema da paciente, que implicou importante desafio à equipe durante o processo de diagnóstico. $\mathrm{O}$ atendimento ocorreu no período de fevereiro a junho de 2006. No início desse atendimento, foram coletadas informações advindas dos prontuários da paciente do Setor de Reumatologia - onde foi diagnosticada a síndrome de Cogan. No Centro de Deficiência Auditiva, realizaram-se os exames audiométricos, sendo Márcia, posteriormente, encaminhada para protetização no Núcleo Integrado de Assistência, Pesquisa e Ensino em Audição (NIAPEA).

Já no Ambulatório de Investigação da Linguagem nos Distúrbios de Audição, local no qual se realizou este atendimento, a equipe complementou dados referentes ao diagnóstico interdisciplinar, nas áreas de Fonoaudiologia e Psicologia. O relato de caso foi estruturado a partir do histórico clínico de Márcia e dos registros das atividades avaliativas desenvolvidas pelos profissionais desse Ambulatório.

\section{Contextualização clínica}

A paciente estudada tinha 27 anos, casada, completou o ensino médio e trabalhava como auxiliar administrativa até se aposentar. Aos 24 anos, quando estava grávida de sua única filha, apresentou vermelhidão nos olhos e procurou o pronto-socorro próximo de sua residência, onde foi diagnosticada com herpes. No ano seguinte, voltou a procurar um serviço de saúde pública, devido

** Pseudônimo utilizado para preservar a identidade da paciente. 
à irritação nos olhos, quando, então, desconfiaram de que pudesse ser alguma síndrome, por isso foi encaminhada ao Hospital São Paulo. Como apresentava sensação de ouvido tampado, fez uma audiometria tonal liminar, na qual se observaram limiares auditivos dentro dos padrões de normalidade. Aos 26 anos, Márcia foi diagnosticada com síndrome de Cogan pelo Setor de Reumatologia do Hospital São Paulo, onde faz acompanhamento até o momento. Começou, então, a partir desse período, a tomar corticóide; teve depressão e apresentou alterações visuais bilaterais. Nesse mesmo ano, passou a apresentar zumbido de pitch grave, bilateral, além de vertigem. Teve um quadro de surdez súbita bilateral. A audiometria tonal liminar revelou uma deficiência auditiva neurossensorial (DANS) de grau severo à orelha esquerda e profundo à direita. Houve rápida piora dos limiares auditivos, bilateralmente. Atualmente, a paciente apresenta anacusia bilateral. No exame otoneurológico, foi observada síndrome periférica, com dificuldades no equilíbrio. Além disso, devido ao comprometimento visual, a paciente necessita andar sempre acompanhada. Recentemente, foi atendida no Núcleo Integrado de Assistência, Pesquisa e Ensino em Audição (NIAPEA) do Hospital São Paulo, participando do processo de seleção e adaptação de Aparelhos de Amplificação Sonora Individual (AASI). Está adaptada com dois AASI, modelo Phoenix 303 da marca Siemens ${ }^{\circledR}$, adequados ao seu grau de perda auditiva, porém com benefícios irrisórios.

\section{Avaliação interdisciplinar dinâmica}

No Ambulatório, durante o atendimento, observou-se que possuía pouca habilidade de leitura orofacial, compreendendo com mais facilidade apenas sua filha, de três anos. Seus familiares retardavam a fala, na tentativa de que Márcia os entendesse; entretanto, esse procedimento dificultava ainda mais a compreensão.

A partir dos relatos, foi possível notar que a família de Márcia a tratava como incapaz; deixavam de conversar com ela, pedindo que fosse descansar quando estava agitada e triste por não conseguir participar, efetivamente, da dinâmica familiar. $\mathrm{O}$ fato de ter se aposentado, deixando de trabalhar em virtude da doença, contribuiu sobremaneira para o sentimento de menos valia de Márcia. Isso foi percebido pela própria paciente, que referiu sentimento de vazio inexplicável por não ter mais o trabalho do qual tanto gostava. A paciente tornou-se totalmente dependente dos familiares para se comunicar, entristecendose consideravelmente, denotando autoconceito cada vez mais depreciativo e afastando-se do convívio com as pessoas.

Após a constatação das dificuldades auditivas e comunicativas de Márcia, bem como a análise do contexto sociofamiliar no qual estava inserida, foi proposto o encaminhamento para a equipe de implante coclear, uma vez que com os AASI a paciente não possuía percepção de fala.

Tendo em vista os relatos de Márcia sobre suas dificuldades e angústias, e mediante a constatação, pela equipe, de suas necessidades, propôs-se orientação familiar e treino de leitura orofacial, além de encaminhamento ao Grupo Brasil***, pois a síndrome de que Márcia é portadora avança para alteração visual significativa, que, no futuro, pode impedir a leitura orofacial.

O treino de leitura orofacial foi realizado pela equipe do Ambulatório, por meio de encontros semanais concomitantes à orientação aos familiares. Esta teve como objetivo descobrir formas mais adequadas de comunicação com Márcia, procurando estimulá-la e conscientizá-la de seu potencial. Foi sugerido à família que mantivesse a fala em velocidade normal, sem exageros na articulação. Além disso, frisou-se a importância de incluir Márcia nas atividades familiares e cotidianas, inserindo-a nas conversas e em sua dinâmica, pois relatos indicam que, antes da doença, a paciente tinha personalidade expansiva, prezando a interação social, aspecto que deve ser constantemente estimulado na vida de Márcia.

O diagnóstico, acompanhado das orientações, foi realizado em dez sessões. Nesse curto período de tempo, notou-se melhora significativa na capacidade de Márcia de se comunicar e interagir socialmente. Atualmente, consegue se comunicar utilizando leitura orofacial e só necessita de acompanhante devido à alteração visual. Os familiares estão cientes das dificuldades da paciente e tentam integrá-la da melhor forma possível aos diversos contextos sociais vivenciados pela família. Do ponto de vista psicológico, a paciente apresenta evolução positiva no autoconceito, sentindo-se mais confiante e capaz de cuidar da própria filha. A paciente ressalta que as orientações recebidas no processo de diagnóstico ambulatorial foram decisivas para se conscientizar de suas dificuldades. Relata que as conversas com a equipe foram estimulantes, ajudando-a na busca de novas soluções e rumos para seus anseios. Como corre o risco de perder a visão, Márcia está aprendendo uma forma de comunicação alternativa em uma organização não-governamental para surdos/cegos, convivendo com outras pessoas com problemas semelhantes aos seus.

\section{DISCUSSÃO}

Um dos primeiros aspectos a serem discutidos refere-se à escuta clínica dos profissionais que atuaram nesse caso, como intérpretes, mantendo a escuta ativa e intervindo nos conflitos biopsíquicos manifestados no discurso da paciente ${ }^{(14)}$. Aliada à escuta cabe aqui ressaltar a postura de acolhimento por parte da equipe, sendo solidária ao sofrimento trazido pela pessoa, por um lado, e atenta às possibilidades de desenvolvimento, por outro. Na maioria dos casos que passam pelo Ambulatório para avaliação, sendo identificada perda auditiva, são indicadas próteses auditivas visando auxiliar a audição. No caso de Márcia, os aparelhos testados não surtiram efeito significativo; por isso, foi necessário pensar, com a equipe, estratégias que pudessem beneficiá-la em seu processo comunicativo. Para tanto, durante a avaliação dinâmica, optou-se por intervenções interativas, priorizando-se o treino orofacial e a orientação aos familiares.

A pessoa com deficiência se beneficia consideravelmente da interação com o meio circundante, pois, se for banida do universo cultural, poderá desenvolver de forma insuficiente

*** O Grupo Brasil é uma organização não-governamental que atende pessoas com surdocegueira, visando melhorar sua qualidade de vida. 
suas funções psicológicas superiores, uma vez que a troca afetiva é imprescindível para a comunicação e a constituição psíquica do indivíduo ${ }^{(7)}$.

A inserção em uma proposta diagnóstica dinâmica que valorize a interação entre o avaliador e o avaliando oferece uma visão mais otimista do que normalmente se constata nas avaliações estáticas ${ }^{(13)}$. Ou seja, motiva o paciente e seus familiares a assumirem uma posição ativa, potencializandose, assim, a capacidade de enfrentamento do problema, posto que a direção do processo não fica exclusivamente centrada no profissional.

Quando chegou ao Ambulatório, Márcia queixava-se: "Ninguém mais liga para mim, sinto-me sozinha, isolada do mundo. Fico desesperada por não ouvir e não entender nada do que querem me dizer". O distanciamento das pessoas das quais ela gostava a desesperava; além disso, sentia-se incapaz de cuidar da filha. Ao trabalhar com os familiares, a equipe mostrou-lhes a necessidade de acolhimento e compreensão da angústia, ansiedade, insegurança e das dificuldades de comunicação vivenciadas por Márcia. O foco das intervenções não se limitou ao treino de comunicação - ao contrário, de maneira mais ampla e interativa, buscou contribuir para o fortalecimento dos vínculos afetivos e da auto-estima da paciente. A humanização no atendimento, devendo o vínculo entre equipe e paciente ser sempre valorizado, com o intuito de oferecer novos padrões de qualidade de vida ${ }^{(4)}$.

O apoio da família é essencial à formação do autoconceito positivo $^{(12)}$ e foi apostando nesse pressuposto que a equipe buscou fortalecer a família, para que Márcia se sentisse aceita, apesar de suas novas limitações sensoriais. Ao final das intervenções, mesmo não tendo a audição melhorada com o uso de aparelhos, Márcia desenvolveu outra forma de comunicação: "Agora consigo entender melhor minha família. Eles não me mandam mais ir dormir por eu estar fora do assunto; entendo praticamente tudo e dou minhas opiniões".

Assim, como já referido, o autoconceito é fator determinante na vida dos indivíduos e influencia todas as suas escolhas ${ }^{(11)}$. Portanto, como o autoconceito depende peremptoriamente da relação com o outro e do sentimento de identidade, a equipe sugeriu a inclusão de Márcia em um grupo de surdos/cegos, buscando novas perspectivas de identificação social.

Gostaríamos de destacar alguns fatores dinâmicos da equipe que julgamos ter contribuído para o sucesso do caso. Em primeiro lugar, destacamos as estratégias de atendimento, que priorizam caso a caso, sempre embasadas em estudos e pesquisas atuais, o que proporciona contínuo aprimoramento da equipe. Todos os casos atendidos no Ambulatório são discutidos entre os profissionais, sob criteriosa supervisão de profissional que, com sua experiência na área, promove uma visão interdisciplinar entre os acadêmicos, o que repercute favoravelmente na evolução dos pacientes. Essa visão baseiase nos princípios da integralidade, equidade e participação do usuário, enfatizando novos espaços de trabalho que sejam criativos, humanos e menos alienadores ${ }^{(4,15)}$.

Nesse sentido, é importante destacar o papel do tutor, isto é, da supervisora e orientadora da equipe, cuja postura de escuta e a forma como favorece o compartilhamento de opiniões tornam-se essenciais para estimular o mesmo comportamento entre os demais profissionais. Assim, nesse serviço ambulatorial, dentro de um hospital/universidade públicos, o clima de confiança que permeia a dinâmica da equipe, bem como o espírito investigativo favorecem a troca de conhecimentos, possibilitando ganhos mútuos, tanto para os acadêmicos em formação quanto para a população atendida.

\section{COMENTÁRIOS FINAIS}

Durante o processo de diagnóstico, mesmo realizado em curto período de tempo, é possível fazer intervenções concomitantes à avaliação que tragam importantes benefícios aos pacientes, contribuindo para operar mudanças significativas no plano comportamental e emocional, visando melhor adaptabilidade social da pessoa. Essa atitude da equipe demonstra superação da condição de atendimento passivo - avaliar para intervir. Logo, vale considerar que os efeitos da avaliação clínica dinâmica dependem não somente do cabedal de conhecimentos específicos, mas também da postura acolhedora e ativa dos profissionais em relação aos pacientes e seus familiares, levando-os por meio de um breve processo de atendimento a uma condição de vida melhor.

\begin{abstract}
The aim of this study was to describe the effects of the interdisciplinar dynamic clinical assessment in a case attended at the Research Ambulatory of Language in Hearing Disorders of the São Paulo Hospital - Federal University of São Paulo. It is describe the case of a 26-year-old woman who presented sudden bilateral deafness, as a result of Cogan's syndrome - which also affects the vision. When the intervention process began, the patient had restricted orofacial reading, difficulty to communicate with her family, social isolation and depressive symptoms. The dynamic assessment based on interactivity and humanistic listening provided conditions for the patient to improve her communication, self-concept and social interaction. From this perspective, it was noticed that, with only ten sessions of intervention, therapeutic effects were operated, therefore breaking the classic assessment/intervention dichotomy at different moments.
\end{abstract}

Keywords: Deafness; Rehabilitation of hearing impaired; Language; Patient care team; Case reports 


\section{REFERÊNCIAS}

1. Brasil. Ministério da Saúde. A pessoa com deficiência e o sistema único de saúde [Internet]. Brasília: Ministério da Saúde; 2006. A pessoa com deficiência auditiva; [citado 2008 Abr 22]. Disponível em: http://bvsms. saude.gov.br/bvs/publicacoes/pessoa_deficiencia.pdf

2. Roslyng-Jensen AM. Importância do diagnóstico precoce na deficiência auditiva. In: Lopes Filho O, editor. Tratado de fonoaudiologia. São Paulo: Roca; 1997. p. 297-309.

3. Imboden JB, Hellmann DB, Stone JH. Current R. Reumatologia: diagnóstico e tratamento. 2a ed. São Paulo: McGraw-Hill. 2008. p. 3324.

4. Goulart BNG, Chiari BM. Avaliação clínica fonoaudiológica, integralidade e humanização: perspectivas gerais e contribuições para reflexão: [revisão]. Rev Soc Bras Fonoaudiol. 2007;12(4):335-40.

5. Luria AR. Pensamento e linguagem: as últimas conferências de Luria. Porto Alegre: Artmed; 1986.

6. Vygotsky LS. Teoria e método em psicologia. São Paulo: Martins Fontes; 1996.

7. Vygotsky LS. Fundamentos de defectologia: obras completas. 2a ed. Havana: Editorial Pueblo e Educación; 1997.

8. Silva MCP, Stefanelli MC. Experiência de uma equipe de saúde mental comunitária na assistência de famílias convivendo com a depressão. Fam Saude Desenvolv. 2002;4(2):89-97.
9. Chiari BM, Bragatto EL, Nishihata R, Carvalho CAF. Perspectivas da atuação fonoaudiológica diante do diagnóstico e prognóstico da surdocegueira. Distúrb Comun. 2006;18(3):371-82.

10. Prioste C, Raiça D, Gomes-Machado ML. Dez questões sobre educação inclusiva da pessoa com deficiência mental. São Paulo: Avercamp; 2006.

11. Zugliani AP, Motti TFG, Castanho RM. O autoconceito do adolescente deficiente auditivo e sua relação com o uso do aparelho de amplificação sonora individual. Rev Bras Educ Espec. 2007;13(1):95-110.

12. Gomes-Machado ML. Deficiência mental: conduta familiar ambivalente no processo de inclusão profissional. Temas Desenvolv. 20052006;14(83-84):63-8.

13. Haywood HC, Tzuriel D. Applications and challenges in dynamic assessment. Peabody J Educ. 2002;77(2):40-63.

14. Cunha MC. Desesquecimento: sobre a escolha do material clínico na pesquisa fonoaudiológica. Distúrb Comun. 2000;12(1):91-9 Casate JC, Corrêa AK. Humanização do atendimento em saúde: conhecimento veiculado na literatura brasileira de enfermagem. Rev Latinoam Enferm. 2005;13(1):105-11. 